\title{
Molecular Modeling and Simulation of Human Stomatin and Predictions for its Membrane Association
}

\author{
Yosuke Kondo ${ }^{1}$, Hideshi Yokoyama ${ }^{1 *}$, Ikuo Matsui ${ }^{2}$ and Satoru Miyazaki ${ }^{1}$
}

${ }^{1}$ Department of Medicinal and Life Science, Faculty of Pharmaceutical Sciences, Tokyo University of Science, Noda, Chiba 278-8510, Japan

${ }^{2}$ Biological Research Institute, National Institute of Advanced Industrial Science and Technology (AIST), 1-1 Higashi, Tsukuba, Ibaraki 305-8566, Japan

"Corresponding author: Hideshi Yokoyama, Department of Medicinal and Life Science, Faculty of Pharmaceutical Sciences, Tokyo University of Science, Noda, Chiba 278-8510, Japan, E-mail: yokoyama@rs.tus.ac.jp

Received date: October 10, 2018; Accepted date: October 24, 2018; Published date: October 29, 2018

Copyright: (c) 2018 Kondo Y, et al. This is an open-access article distributed under the terms of the Creative Commons Attribution License, which permits unrestricted use, distribution and reproduction in any medium, provided the original author and source are credited.

\begin{abstract}
Stomatin is a membrane protein in human red blood cells. The crystal structure, in which the monomeric stomatin from the hyperthermophilic archaeon Pyrococcus horikoshii consists of the $\alpha / \beta$ domain and the C-terminal $\alpha$-helical segment, forms a homo-trimer, and stomatin is organized into further high order homo-oligomeric complexes, comprising 9- to 12-mers. To better understand the molecular functions of stomatin, the hypothesis how human stomatin oligomerizes and is associated with cell membranes should be validated. Here, we report what conformations can be generated from the stomatin structure by estimating the flexibility of a-helical segments of human stomatin. And we also simulate how the oligomeric structure of human stomatin interacts with cell membranes. The results showed that the $\alpha$-helical segments can make flexible movements; the $\alpha$-helical segment and the $\alpha / \beta$ domain of the monomer can form a flat structure, and the $\alpha$-helical segments of the trimer can approach lipid membranes. Based on the flat structure of human stomatin, we proposed a hypothetical oligomeric model to interact with the surface of cell membranes. The oligomeric model well explains the stomatin functions as a scaffolding protein to support the cell membrane.
\end{abstract}

Keywords: Cell membrane; Lipid rafts; Molecular dynamics simulation; SPFH domain; Stomatin protein

Abbreviations: MD: Molecular Dynamics; R.M.S.D: Root-MeanSquare Deviation; STOPP: Stomatin Operon Partner Protein

\section{Introduction}

Stomatin is one of the major integral membrane proteins of human erythrocytes. The protein is deficient from the erythrocyte membranes of patients with overhydrated hereditary stomatocytosis, a form of hemolytic anemia characterized by stomatocytic red blood cells with abnormal permeability to $\mathrm{Na}^{+}$and $\mathrm{K}^{+}$[1]. This appears to be caused by mis-trafficking of stomatin during erythropoiesis [2]. Human stomatin is ubiquitously expressed in essentially every tissue except brain [3]. It has been reported that stomatin modulates the activity of acid-sensing ion channels [4,5] and GLUT-1 glucose transporter [6,7]. Stomatin is organized into high order homo-oligomeric complexes of approximately $300 \mathrm{kDa}$, comprising 9- to 12-mers [8], and is localized in detergent-resistant membrane domains, which are also termed lipid rafts [9-11]. It has also been reported that stomatin is a major component of vesicles produced by red cells [12], and potentiates cell fusion [13]. Recently, mutational studies of stomatin have been reported to elucidate subcellular localization, oligomerization, and biochemical properties [14]. Stomatin-like proteins have been found in most species of eukaryotes, bacteria, and archaea [15], and the related proteins are known as stomatin, prohibitin, flotillin, and $\mathrm{HflK} / \mathrm{C}$ (SPFH) domain proteins which are found in the lipid rafts of various cellular membranes [15-17]. These SPFH domain proteins could function as a membrane-bound oligomeric scaffolding protein in lipid rafts [10], in the topologically similar manner to caveolins [18].
Our group previously determined the first crystal structure of the core domain of stomatin from the hyperthermophilic archaeon Pyrococcus horikoshii (ph) [19]. In this structure, the SPFH domain was found to form a stable trimer, while three C-terminal $\alpha$-helical segments extended from the apexes of the triangle. The crystal structures of the mouse stomatin-domain were shown to assemble into a banana shaped dimer [20]. As an overall structure of human stomatin has not been determined, it remains to be elucidated how stomatin proteins oligomerize for themselves, and how they are associated with the membranes. Here, we report the molecular modeling and simulation of human stomatin using the structures determined previously as starting models. And we also validate the flexibility of C-terminal $\alpha$-helical segments of stomatin, and estimate the interactions between stomatin and lipid molecules. These results would suggest the hypothesis how stomatin oligomerizes and is associated with membranes.

\section{Materials and Methods}

\section{Modeling of the 3D structure of human stomatin}

We obtained the amino acid sequence of human stomatin from the RefSeq database at NCBI (http://www.ncbi.nlm.nih.gov/refseq/, accession: NP 004090). To determine template structures of human stomatin, we used a BLAST algorithm [21] in Discovery Studio 2016 [22] for searching homologous sequences of human stomatin in Protein Data Bank (PDB) [23]. The BLAST search found Pyrococcus horikoshii stomatin (PDB code: 3BK6) [19] and mouse stomatin (PDB code: 4FVG) [20]. We aligned the amino acid sequences of stomatins in Discovery Studio (Align sequences or sequence profiles protocol [24]). Based on the multiple sequence alignment, 3D models of human stomatin were constructed in Discovery Studio (Homology Modeling 
protocol [25]). The modeled structure was verified by Verify Protein protocol [26] and Ramachandran plot [27] in Discovery Studio.

\section{Modeling of the 3D structures of trimeric stomatin and lipid membranes}

We created the trimeric structure of human stomatin by superimposing to the asymmetric unit of ph stomatin [19]. The modeled structure of human stomatin was superimposed on the chains A, B, and C of ph stomatin in Discovery Studio (Superimpose Proteins protocol). To predict membrane association of the trimeric structure of human stomatin, we used CHARMM-GUI Membrane Builder [28] to build the structure of lipid bilayer membranes, which consists of 600 POPC (1-palmitoyl-2-oleoyl-sn-glycero-3-phosphocholine) and 600 cholesterol molecules. The trimeric structure of human stomatin was put on the surface of the lipid membranes in Discovery Studio.

\section{MD simulation}

We used the AMBER16 software [29] for molecular dynamics (MD) simulation accelerated by single GPU (GeForce GTX-1080). The AMBER 14sb and lipid 14 force fields [30,31] were allocated to the structures of human stomatin and lipid membranes, respectively. We created a rectangular box around the structure of stomatin and lipid membranes and filled with TIP-3P water molecules [32]. We put counter ions in the box not to have any electric charges in the system. Energy minimization was performed to the system by the steepest descent method (10,000 steps) and conjugate gradient method (10,000 steps). $1 \mathrm{~ns}$ MD simulations were conducted by gradually increasing the reference temperature from $0 \mathrm{~K}$ to $310 \mathrm{~K}$ by Langevin dynamics $(\mathrm{ntt}=3$, gamma $\ln =1.0$ ). The time step was set to $2 \mathrm{fs}$ by SHAKE algorithm of hydrogen atoms (ntc=2) [33]. The cutoff of van der Waals energy was set to $10 \AA ̊$. The Coulomb energy was computed by particle mesh Ewald (PME) algorithm [34]. 200 ns MD simulations were conducted with constant pressure condition $(n t b=2)$, and the reference pressure was set to $1 \mathrm{~atm}$. We conducted ten independent energy minimizations and MD simulations by changing the initial structure of the monomeric structure of human stomatin or the structure of the trimeric stomatin and lipid membranes.

\section{Analysis of MD trajectories}

We used $\mathrm{C}-\mathrm{N}$ bonds in peptide backbones for defining an axis of a peptide sequence. By summing vectors from the $\mathrm{C}$ atom to the $\mathrm{N}$ atom, the directional vector of the peptide sequence was obtained. The angle between two vectors of peptide sequences was calculated from each conformation in MD trajectories. To define the plane of a lipid membrane, we conducted a principal component analysis for each structure of the lipid membranes in MD trajectories. The angle between the vertical vector of a membrane plane and the vector of a peptide sequence in stomatin was computed.

\section{Results}

\section{Modeling of the structure of human stomatin}

The 3D structure of human stomatin has not been experimentally determined. In this study, homology modeling was employed to obtain the 3D model of human stomatin. The homology search tool found two stomatins determined by X-ray crystallography. One of the crystal structures is ph stomatin. This crystal structure has the $\alpha / \beta$ core domain and the C-terminal $\alpha$-helical segment $(\alpha 5+$ partial $\alpha 6)$ of ph stomatin [19]. The template structure is corresponding from Pro85 to Thr254 in human stomatin (Figure 1). The other crystal structure is mouse stomatin. This crystal structure has the $\alpha / \beta$ core domain of mouse stomatin [20]. The template structure is corresponding from Val93 to Gln201 in human stomatin. The sequence identity between human (P85-T254) and ph (P55-T224) stomatins is 48.2\%, while that between human (V93-Q201) and mouse (V94-Q202) stomatins is 96.3\%. The mouse template structure has a higher sequence identity than ph stomatin but lacks some residues in the $\mathrm{N}$-terminal or $\mathrm{C}$ terminal regions compared with ph stomatin. Meanwhile, the BLAST search found some template structures corresponding to the $\mathrm{N}$ terminal or C-terminal region of human stomatin. The e-values of the template structures were larger than 1 . These values were very larger than that of ph stomatin $\left(6.0 \times 10^{-67}\right)$ or mouse stomatin $\left(1.8 \times 10^{-53}\right)$. This shows that the template structures are not appropriate to build the N-terminal (A1-L84) and C-terminal (L255-G287) regions of human stomatin.

The 3D structure of human stomatin (P85-T254) was modeled (Figure $2 \mathrm{~A}$ ), and the modeled structure of human stomatin has $\alpha 1-\alpha 6$ helices and $\beta 2-\beta 5$ strands, which are named after helices and strands in ph stomatin [19]. The verify scores are relatively higher in the $\alpha 3$ and $\alpha 4$ helices and $\beta 4$ and $\beta 5$ strands, and they are relatively lower in the $\alpha 1, \alpha 2, \alpha 5$ and $\alpha 6$ helices and $\beta 2$ and $\beta 3$ strands (Figures $2 \mathrm{~A}$ and $2 \mathrm{~B}$ ). The sum of verify scores in the modeled structure of human stomatin was 51.5. The verify expected high and low scores were 77.0 and 34.7, respectively. The verify score of the modeled stomatin is smaller than the verify expected high score and larger than the verify expected low score. The Ramachandran plot shows that $99.4 \%$ (169/170) residues are included in the favorable or allowed regions, and K194 is an only residue in the disallowed region (Figure 2C).

\section{Simulation of the monomer of human stomatin}

MD simulations of the monomeric structure of human stomatin show a variety of movements of the $\alpha 5$ helix. To analyze the relative position of the $\alpha$-helical segment and the $\alpha / \beta$ core domain, the angles of axes of $\alpha 5$ helix and $\alpha 2$ or $\alpha 4$ helix were calculated (Figures $3 \mathrm{~A}$ and $3 \mathrm{~B})$. The results show that the angle between $\alpha 2$ and $\alpha 5$ helices can approximately move from $30^{\circ}$ to $140^{\circ}$ in the $200 \mathrm{~ns} \mathrm{MD}$ simulations, and the angle between $\alpha 4$ and $\alpha 5$ helices can approximately move from $10^{\circ}$ to $120^{\circ}$ in the $200 \mathrm{~ns} \mathrm{MD}$ simulations. The movements of the monomeric structure showed conformations in which each $\alpha$-helical segment moves to almost the same plane of the $\alpha / \beta$ core domain (Figure 4).

\section{Simulation of the trimer of human stomatin and lipid membranes}

MD simulations of the trimeric structure of human stomatin and lipid membranes also show a variety of movements of the a 5 helix. The angles of the $\alpha$-helical segment and the plane of the lipid membrane show that the $\alpha$-helical segment can approximately move from $0^{\circ}$ to $80^{\circ}$ in the $200 \mathrm{~ns} \mathrm{MD}$ simulations (Figure 5). The result shows that the movements of the $a 5$ helix and lipid membranes can move almost any directions. These movements showed that the $\alpha$-helical segments move close to the plane of the lipid membrane (Figure 6). 


\section{Discussion}

The obtained model of human stomatin consists of the $\alpha / \beta$ domain and the $\alpha$-helical segment. The $\alpha / \beta$ domain and $\alpha$-helical segment is estimated to be located on cell membranes in cytosol. Although the modeled structure does not have $\mathrm{N}$-terminal and $\mathrm{C}$-terminal regions of human stomatin, the $\mathrm{N}$-terminal region is predicted to have transmembrane regions, and the C-terminal region is essential to oligomerize stomatins. Appropriate template structures of the $\mathrm{N}$ terminal and C-terminal regions of human stomatin did not exist because the template structures of the terminal regions have larger evalues compared with ph stomatin and mouse stomatin. It is possible to be a bad accuracy in predicting the terminal regions, even if we used the template structures. In addition, terminal regions of proteins are often disordered. It would be difficult to build the terminal regions of human stomatin. The results of the Ramachandran plot and Verify Protein (Figure 2) suggest that the modeled structure is accurate to a certain extent. However, some residues in the modeled structure have low verify scores. Residues with low verify scores show that there are hydrophobic patches on the surface of the structure. The result suggests that the hydrophobic patches of human stomatin are responsible for binding to lipid membranes.

The crystal structure of ph stomatin was determined as a homotrimer [19]. In the asymmetric unit of the crystal, the a-helical segment is flexible because three $\alpha$-helical segments are not same positions compared with each of the $\alpha / \beta$ domains [19]. The MD simulations were conducted in an explicit solvent condition to analyze the movements in water solvent. The MD simulations showed that the $\alpha$-helical segment can make flexible movements because the relative position between the $\alpha / \beta$ domain and $\alpha$-helical segment was flexible (Figure 3). However, the $\alpha$-helical segment cannot move any directions. These results suggest that the conformation of the $\alpha / \beta$ domain and the $\alpha$-helical segment is flexible but slightly limited. The conformations include a movement that the $\alpha$-helical segment moves in a same plane of the $\alpha / \beta$ domain. The result suggests that the $\alpha$ helical segment can make a conformation interacting with cell membranes.

MD simulations of the trimeric structure were conducted to be supposed in the situation that the trimer is associated with cell membranes. The results showed that the relative positions of the $\alpha$ helical segments and the planes of the lipid membranes are not limited strictly. Therefore, the movements of a-helical segments may not depend on the lipid membrane. The movements showed that the $\alpha$ helical segments can move close to the lipid membrane and stabilize on it. The result suggests that the $\alpha$-helical segments can interact with cell membranes. In order to validate the results of human stomatin, we should perform MD simulations by using the crystal structure of ph stomatin or mouse stomatin with the modeled lipid membranes. However, it took about more than one month to conduct the $200 \mathrm{~ns}$ MD simulation of the modeled structure of human stomatin with the lipid membranes in our computing environment. We try to perform the MD simulations of $\mathrm{ph}$ and mouse stomatin in future works to simulate whether ph stomatin and mouse stomatin have a same function as of human stomatin.

Cell membranes change their shapes during processes such as division, membrane fusion, vesicle trafficking, and so on. These processes are accompanied by membrane curvature, which can be modulated by changes in the oligomerization of curvature scaffolding proteins $[35,36]$. Caveolins, one of these scaffolding proteins, show common topological properties with SPFH domain proteins such as stomatin and flotillin [18]. The $a$-helical segments of stomatin may flexibly move along with the membrane surface as described above. This movement may cause membrane bending. It remains to be elucidated whether the basic unit of human stomatin is a trimer. In the structure of the mouse stomatin-domain [20], each $\alpha / \beta$ domain assembles into a dimer; and the dimer is a basic unit. Here in our report, we hypothesize that human stomatin assembles into a trimer as a basic unit, and the trimeric model can explain well that stomatin is associated with cell membranes.

A C-terminal hydrophobic region (264STIVFPLPI272) of human stomatin is crucial for oligomerization [11]. Based on the secondarystructure prediction program PSIPRED [37], the region is between $a 6$ (residues 244-259) and $\alpha 7$ (residues 271-281). Given that $\alpha 6$ and $\alpha 7$ helices form a helix bundle in an anti-parallel way, the region of the hairpin loop between $\alpha 6$ and $\alpha 7$ will interact with that of another molecule, and the oligomers may be formed via the interaction. According to these points, we propose a hypothetical oligomerized 9mer model of human stomatin (Figure 7). This model supports the previous report that stomatin is organized into oligomeric complexes of 9- to 12-mers [8]. Stomatin functions as a scaffolding protein to support the cell membrane from underneath as discussed in Umlauf et al. [10]. Now, we try to determine the overall structure of ph stomatin including N-terminal membrane-spanning and C-terminal hydrophobic regions to elucidate such oligomerization mechanisms in detail [38].

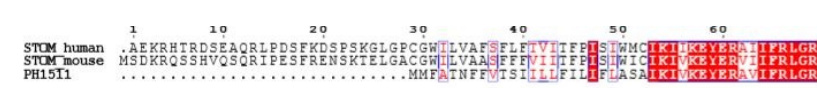

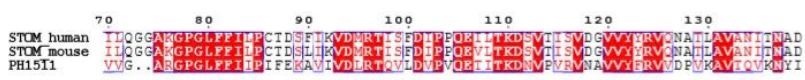

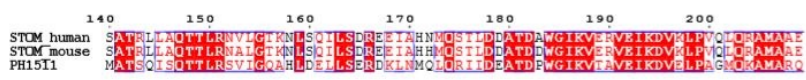
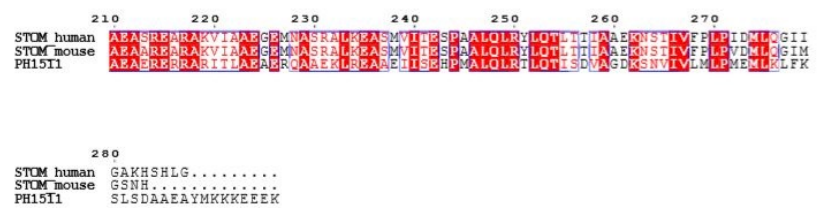

Figure 1: The multiple sequence alignment of human stomatin (STOM human), mouse stomatin (STOM mouse), and Pyrococcus horikoshii stomatin (PH1511). The sequences were obtained from the RefSeq database at NCBI (STOM human, NP 004090; STOM mouse, NP 038543; PH1511, WP 010885588). The sequences were aligned with ClustalW [24] and displayed by ESPript [38]. A black dot above the sequences marks every 10 amino acid residues. White lettering boxed with a red background indicates residues that are conserved in all sequences, and red lettering indicates similar residues. 
Citation: Kondo Y, Yokoyama H, Matsui I, Miyazaki S (2018) Molecular Modeling and Simulation of Human Stomatin and Predictions for its Membrane Association. J Data Mining Genomics Proteomics 9: 216. doi:10.4172/2153-0602.1000216

Page 4 of 6

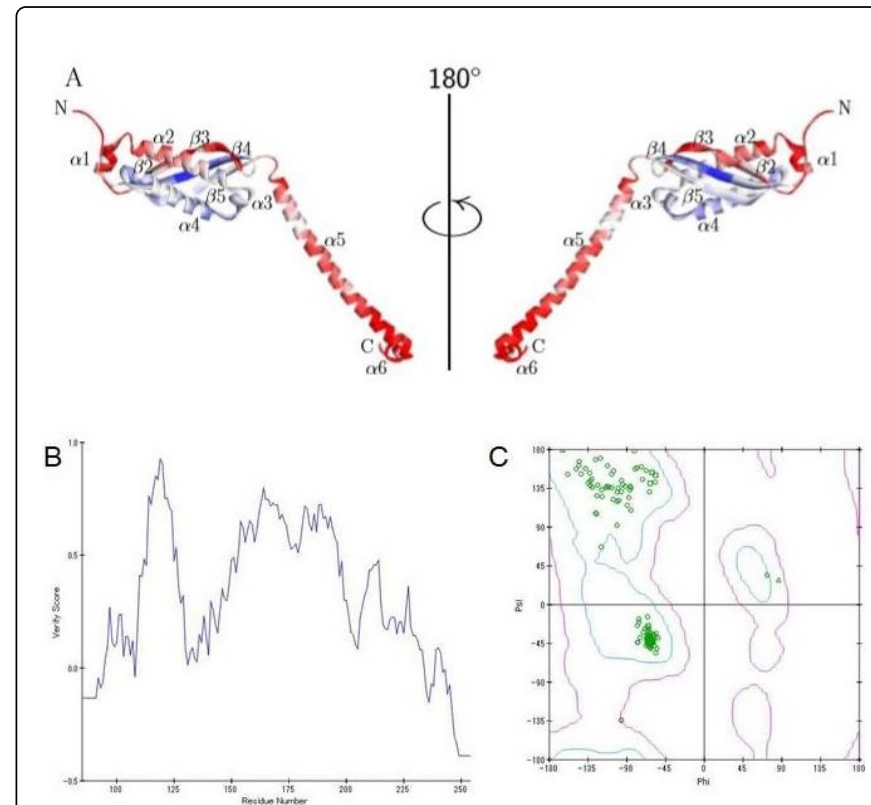

Figure 2: (A) The 3D model of human stomatin. The residues were colored by blue-white-red. The residues with relatively high verify scores were shown in blue, those with intermediate verify scores were shown in white, and those with relatively low verify scores were shown in red. (B) Scores of Verify 3D for each residue. (C) Ramachandran plot. The inner sides of the cyan lines indicate favorable regions, and those of the magenta lines indicate allowed regions. The outer sides of the magenta lines indicate disallowed regions. The triangles displayed Gly, squares displayed Pro, and circles displayed the other amino acids. The residues in favorable or allowed regions were shown in green, and those in disallowed regions were shown in red.
A

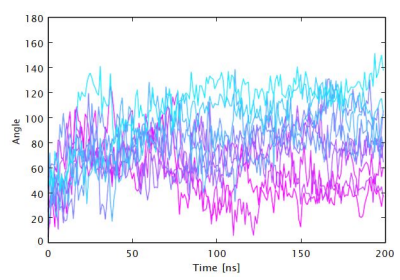

B

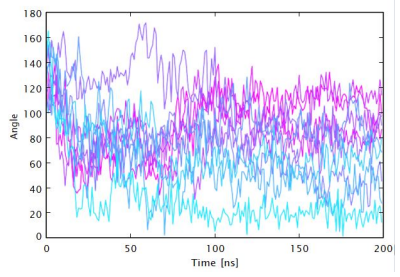

Figure 3: (A) Time variations of an angle between $\alpha 2$ and $\alpha 5$ helices of human stomatin. (B) Time variations of an angle between $\alpha 4$ and $\alpha 5$ helices of human stomatin. We conducted $10 \mathrm{MD}$ simulations of monomeric human stomatin. The curves colored from cyan to magenta indicate each of the $10 \mathrm{MD}$ trajectories, including the angle between the two helices.

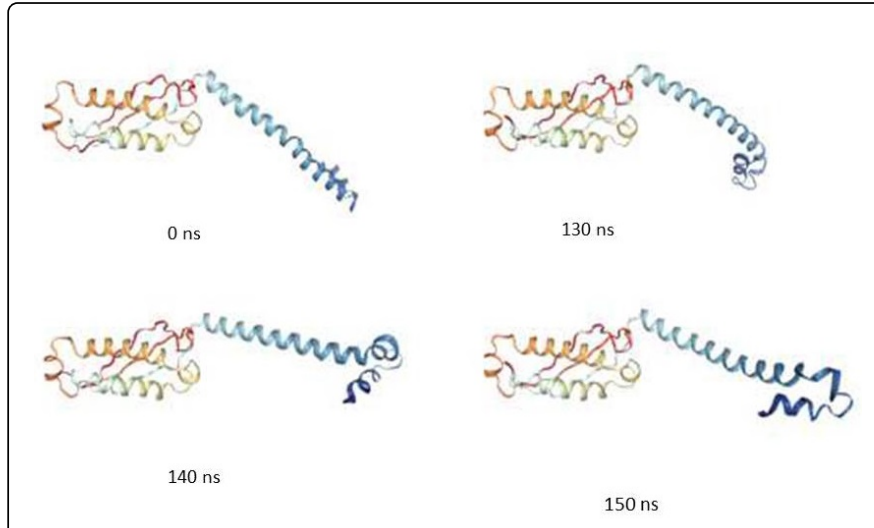

Figure 4: The monomeric structure of human stomatin in $\mathrm{MD}$ simulation. The residues from $\mathrm{N}$ - to $\mathrm{C}$-terminus were colored from warm to cool colors.

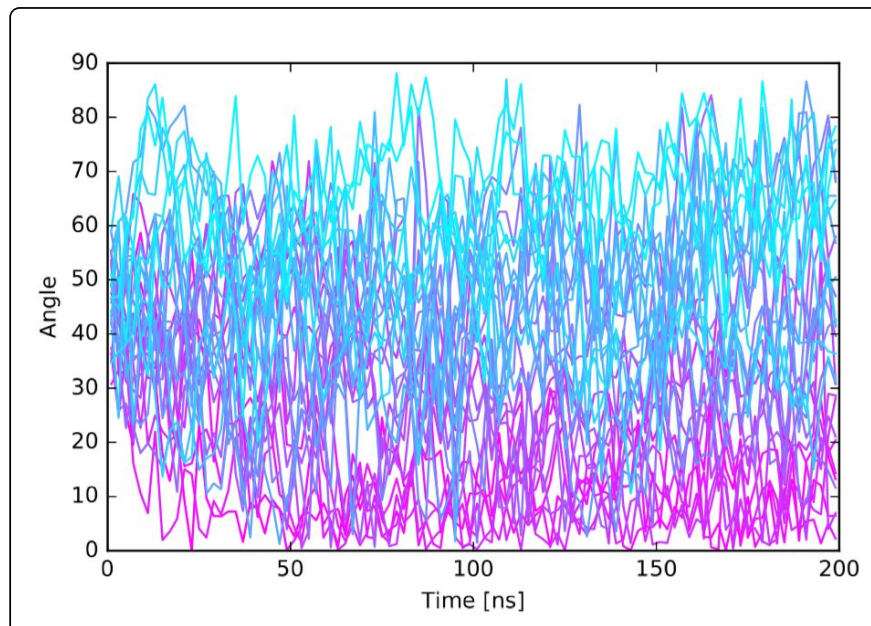

Figure 5: Time variations of an angle between an $\alpha 5$ helix of human stomatin and a plane of lipid membranes. We conducted $10 \mathrm{MD}$ simulations of trimeric human stomatin with modeled lipid membranes. The curves colored from cyan to magenta indicate each of the $30 \mathrm{MD}$ trajectories, including the angle between each of the three $a 5$ helices and a plane of the lipid membranes. 
Citation: Kondo Y, Yokoyama H, Matsui I, Miyazaki S (2018) Molecular Modeling and Simulation of Human Stomatin and Predictions for its Membrane Association. J Data Mining Genomics Proteomics 9: 216. doi:10.4172/2153-0602.1000216

Page 5 of 6
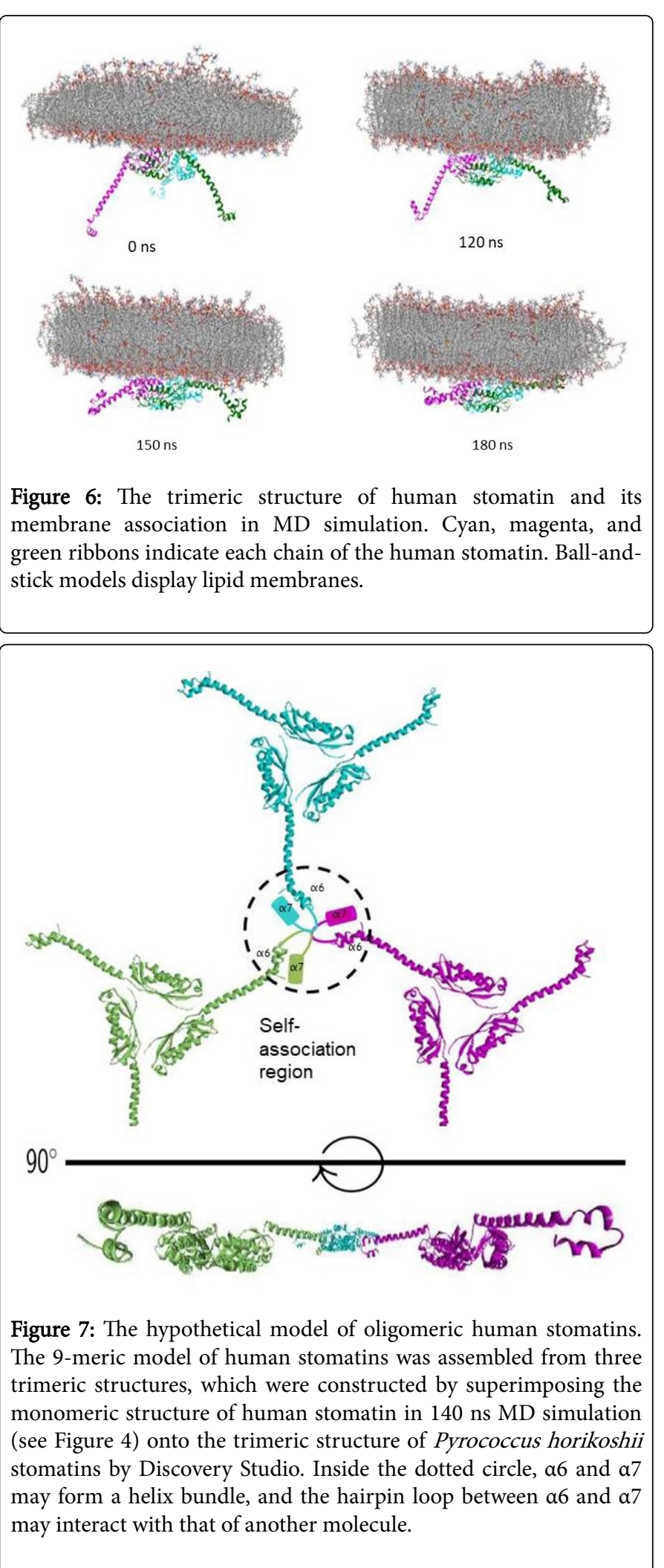

\section{Conflict of Interest}

The authors declare no conflict of interest.

\section{Acknowledgement}

This work was supported in part by JSPS KAKENHI Grant Numbers 264403344 and 17K07316 to H.Y. from the Ministry of Education, Culture, Sports, Science and Technology (MEXT).

\section{References}

1. Stewart GW, Argent AC, Dash BCJ (1993) Stomatin: A putative cation transport regulator in the red cell membrane. Biochim Biophys Acta 1225: $15-25$.

2. Fricke B, Parsons SF, Knöpfle G, von Düring M, Stewart GW (2005) Stomatin is mis-trafficked in the erythrocytes of overhydrated hereditary stomatocytosis, and is absent from normal primitive yolk sac-derived erythrocytes. Br J Haemratol 131: 265-277.

3. Gallagher PG, Forget BG (1995) Structure, organization, and expression of the human band $7.2 \mathrm{~b}$ gene, a candidate gene for hereditary hydrocytosis. J Biol Chem 270: 26358-26363.

4. Price MP, Thompson RJ, Eshcol JO, Wemmie JA, Benson CJ (2004) Stomatin modulates gating of acid-sensing ion channels. J Biol Chem 279: 53886-53891.

5. Wetzel C, Hu J, Riethmacher D, Benckendor A, Harder L, et al. (2007) A stomatin-domain protein essential for touch sensation in the mouse. Nature 445: 206-209.

6. Zhang JZ, Abbud W, Prohaska R, Ismail-Beigi F (2001) Overexpression of stomatin depresses GLUT-1 glucose transporter activity. Am J Physiol Cell Physiol 280: C1277-C1283.

7. Rungaldier S, Oberwagner W, Salzer U, Csaszar E, Prohaska R (2013) Stomatin interacts with GLUT1/SLC2A1, band 3/SLC4A1, and aquaporin-1 in human erythrocyte membrane domains. Biochim Biophys Acta 1828: 956-966.

8. Snyers L, Umlauf E, Prohaska R (1998) Oligomeric nature of the integral membrane protein stomatin. J Biol Chem 273: 17221-17226.

9. Mairhofer M, Steiner M, Mosgoeller W, Prohaska R, Salzer U (2002) Stomatin is a major lipid-raft component of platelet alpha granules. Blood 100: 897-904.

10. Umlauf E, Csaszar E, Moertelmaier M, Schuetz GJ, Parton RG, et al. (2004) Association of stom- atin with lipid bodies. J Biol Chem 279: 23699-23709.

11. Umlauf E, Mairhofer M, Prohaska R (2006) Characterization of the stomatin domain involved in homo-oligomerization and lipid raft association. J Biol Chem 281: 23349-23356.

12. Salzer U, Zhu R, Luten M, Isobe H, Pastushenko V, et al. (2008) Vesicles generated during storage of red cells are rich in the lipid raft marker stomatin. Transfusion 48: 451-462.

13. Lee JH, Hsieh CF, Liu HW, Chen CY, Wu SC, et al. (2017) Lipid raftassociated stomatin enhances cell fusion. FASEB J 31: 47-59.

14. Rungaldier S, Umlauf E, Mairhofer M, Salzer U, Thiele C, et al. (2017) Structure-function analysis of human stomatin: A mutation study. PLoS One 12: e0178646.

15. Tavernarakis N, Driscoll M, Kyrpides NC (1999) The SPFH domain: Implicated in regulating targeted protein turnover in stomatins and other membrane-associated proteins. Trends Biochem Sci 24: 425-427.

16. Browman DT, Hoegg MB, Robbins SM (2007) The SPFH domaincontaining proteins: More than lipid raft markers. Trends Cell Biol 17: 394-402.

17. Lapatsina L, Brand J, Poole K, Daumke O, Lewin GR (2012) Stomatindomain proteins. Eur J Cell Biol 91: 240-245.

18. Bauer M, Pelkmans L (2006) A new paradigm for membrane-organizing and -shaping scaffolds. FEBS Lett 580: 5559-5564.

19. Yokoyama H, Fujii S, Matsui I (2008) Crystal structure of a core domain of stomatin from Pyro-coccus horikoshii illustrates a novel trimeric and coiled-coil fold. J Mol Biol 376: 868-878. 
Citation: Kondo Y, Yokoyama H, Matsui I, Miyazaki S (2018) Molecular Modeling and Simulation of Human Stomatin and Predictions for its Membrane Association. J Data Mining Genomics Proteomics 9: 216. doi:10.4172/2153-0602.1000216

Page 6 of 6

20. Brand J, Smith ES, Schwefel D, Lapatsina L, Poole K, et al. (2012) A stomatin dimer modulates the activity of acid-sensing ion channels. EMBO J 31: 3635-3646.

21. Altschul SF, Madden TL, Schäffer AA, Zhang JH, Zhang Z, et al. (1997) Gapped BLAST and PSI- BLAST: A new generation of protein database search programs. Nucleic Acids Res 25: 3389-3402.

22. Accerlys Inc (2016) Discovery Studio 2016. San Diego, CA.

23. Berman HM, Westbrook J, Feng Z, Gilliland G, Bhat TN, et al. (2000) The protein data bank. Nucleic Acids Res 28: 235-242.

24. Thompson JD, Higgins DG, Gibson TJ (1994) CLUSTAL W: Improving the sensitivity of progres- sive multiple sequence alignment through sequence weighting, position-specific gap penalties and weight matrix choice. Nucleic Acids Res 22: 4673-4680.

25. Sali A, Blundell TL (1993) Comparative protein modeling by satisfaction of spatial restraints. J Mol Biol 234: 779-815.

26. Eisenberg D, Luthy R, Bowie JU (1997) Verify3D: Assessment of protein models with three-dimensional profiles. In: Carter, CW and Sweet, RM, editor, Macromol Crystal, PT B, volume 277 of Methods in Enzymology. pp. 396-404.

27. Lovell SC, Davis IW, Adrendall WB, de Bakker PIW, Word JM, et al (2003) Structure validation by $C \alpha$ geometry: $\varphi, \psi$ and $C \beta$ deviation. Proteins 50: 437-450.

28. Brooks BR, Brooks CL III, Mackerell AD Jr, Nilsson L, Petrella RJ, et al (2009) CHARMM: The Biomolecular Simulation Program. J Comput Chem 30: 1545-1614.

29. Salomon-Ferrer R, Case DA, Walker RC (2013) An overview of the Amber biomolecular simulation package. WIREs Comput Mol Sci 3: 198-210.
30. Maier JA, Martinez C, Kasavajhala K, Wickstrom L, Hauser KE, et al. (2015) 14SB: Improving the accuracy of protein side chain and backbone parameters from 99SB. J Chem Theo Comput 11: 3696-3713.

31. Madej BD, Gould IR, Walker RC (2015) A parameterization of cholesterol for mixed lipid bilayer simulation within the amber lipid14 force field. J Phys Chem B 119: 12424-12435.

32. Jorgensen WL, Chandrasekhar J, Madura JD, Impey RW, Kkein ML (1983) Comparison of simple potential functions for simulating liquid water. J Chem Phys 79: 926-935.

33. Miyamoto S, Kollman P (1992) SETTLE - An analytical version of the SHAKE and RATTLE algorithm for rigid water models. J Comput Chem 13: 952-962.

34. Darden T, York D, Pedersen L (1993) Particle mesh Ewald - an N.log(N) method for Ewald sums in large systems. J Chem Phys 98: 10089-10092.

35. McMahon HT, Gallop JL (2005) Membrane curvature and mechanisms of dynamic cell membrane remodelling. Nature 438: 590-596.

36. Martens S, McMahon HT (2008) Mechanisms of membrane fusion: disparate players and common principles. Nature Rev Mol Cell Biol 9: 543-556.

37. McGuffin LJ, Bryson K, Jones DT (2000) The PSIPRED protein structure prediction server. Bioinformatics 16: 404-405.

38. Gouet P, Courcelle E, Stuart DI, Metoz F (1999) ESPript: Analysis of multiple sequence alignments in PostScript. Bioinformatics 15: 305-308. 